\title{
TRPM2 SNP genotype previously associated with susceptibility to Rhodococcus equi pneumonia in Quarter Horse foals displays differential gene expression identified using RNA-Seq
}

\author{
Cole M. McQueen ${ }^{1}$, Canaan M. Whitfield-Cargile ${ }^{1}$, Kranti Konganti ${ }^{2,4}$, Glenn P. Blodgett ${ }^{3}$, Scott V. Dindot ${ }^{4 *}$ \\ and Noah D. Cohen ${ }^{1 *}$
}

\begin{abstract}
Background: Rhodococcus equi (R. equi) is an intracellular bacterium that affects young foals and immuno-compromised individuals causing severe pneumonia. Currently, the genetic mechanisms that confer susceptibility and/or resistance to R. equi are not fully understood. Previously, using a SNP-based genome-wide association study, we identified a region on equine chromosome 26 associated with culture-confirmed clinical pneumonia. To better characterize this region and understand the function of the SNP located within TRPM2 that was associated with $R$. equi pneumonia, we performed RNA-Seq on 12 horses representing the 3 genotypic forms of this SNP.

Results: We identified differentially expressed genes in the innate immune response pathway when comparing homozygous A allele horses with the AB and BB horses. Isoform analyses of the RNA-Seq data predicted the existence of multiple transcripts and provided evidence of differential expression at the TRPM2 locus. This finding is consistent with previously demonstrated work in human cell lines in which isoform-specific expression of TRPM2 was critical for cell viability.
\end{abstract}

Conclusions: This work demonstrates that SNPs in TRPM2 are associated with differences in gene expression, suggesting that modulation of expression of this innate immune gene contributes to susceptibility to R. equi pneumonia.

Keywords: Rhodococcus equi, Horses, RNA-Seq, Gene expression, Pneumonia

\section{Background}

Rhodococcus equi (R. equi) is a pathogen that predominantly affects young foals causing pneumonia as well as extra-pulmonary disorders [1-5]. Currently, there is no approved vaccine for protection against $R$. equi pneumonia, and other preventative interventions, such as transfusion of hyperimmune plasma, are expensive, labor-intensive, and incompletely effective [4,6]. Isolates of $R$. equi that cause disease in foals must bear the virulence associated

\footnotetext{
* Correspondence: sdindot@cvm.tamu.edu; ncohen@crm.tamu.edu ${ }^{4}$ Department of Veterinary Pathobiology, Texas A\&M University College of Veterinary Medicine \& Biomedical Sciences, College Station, TX, USA 'Department of Large Animal Clinical Sciences, Texas A\&M University College of Veterinary Medicine \& Biomedical Sciences, College Station, TX, USA Full list of author information is available at the end of the article
}

protein A (VapA) gene in a plasmid; however, presence of the plasmid and VapA expression alone is not sufficient to cause disease, indicating that host factors are of great importance $[7,8]$. In addition to anecdotal reports of some mares having multiple infected foals while other mares in the same environment never have an affected foal, several candidate gene studies suggest a genetic basis for $R$. equi susceptibility [9-13]. Because of gene-selection bias and phenotypic misclassification, the associations from candidate gene studies have been weak and potentially biased [14].

Recently, our laboratory identified a region on chromosome 26 associated with $R$. equi pneumonia in a genomewide association study (GWAS) [15] using a commercially 
available single nucleotide polymorphism (SNP) array $[15,16]$. Four SNPs were associated with clinical disease in a region spanning several predicted genes. One of the SNPs was well suited to serve as a marker because it was located within a candidate gene (viz., the transient receptor potential cation channel, subfamily $M$, member2 [TRMP2]) and could be easily and accurately genotyped [15]. This SNP was associated with 3 genotypes designated $\mathrm{AA}, \mathrm{AB}$, and BB alleles. Because SNPs are merely indicators of location and are very rarely actual causal mutations, it remains unclear which genes in this region might explain the observed association of genotype with disease. More importantly, these SNPs alone do not provide any functional information regarding the relationship between genotype and phenotype across this region. The marker SNP in TRPM2 is a synonymous substitution and does not change the amino acid or protein sequence and is thus unlikely to be causally associated with disease. It is biologically plausible, however, that this SNP is a marker for other genetic elements nearby that might regulate gene expression. Thus, other approaches including investigating gene expression are necessary to further understand the observed association of the TRPM2 SNP and $R$. equi pneumonia.

Investigating the whole transcriptome using RNASequencing (RNA-Seq) provides an unbiased approach for gene expression analysis, including the analysis of alternatively spliced transcripts (viz., isoforms), [17] yielding greater possibility for identifying true expression phenotypes associated with the genotype(s) of interest. RNA-Seq can also be used to identify and examine unnanotated genes and isoforms. The principal aims of this study were: 1) to determine whether gene expression (including analysis of isoforms) in the TRPM2 gene region was associated with the marker genotypes for the TRPM2 SNP (i.e., $\mathrm{AA}, \mathrm{AB}$, or $\mathrm{BB}$ alleles), using RNA-Seq (targeted approach); and, 2) to perform associations of gene expression across the transcriptome with the TRPM2 genotypes (untargeted approach). As a secondary aim, we examined the association of gene expression across the transcriptome with history of $R$. equi pneumonia. The objectives of this study were to identify whether the marker genotype identified in TRPM2 was an indicator of differential expression, and to identify any other genes that were differentially expressed between the TRPM2 SNP genotypes. Completing these objectives would allow us to find key biological pathways and processes involved in susceptibility to $R$. equi pneumonia [18-20].

\section{Methods}

\section{Study population}

The study population was derived from the source population for our GWAS described above (viz., foals born during 2011 at the 6666 Ranch) [15], which had a cumulative incidence of $R$. equi pneumonia of 17\% during 2011. Briefly, foals were monitored for clinical signs of fever, cough, nasal discharge, and lethargy which were suggestive of pneumonia. Foals with clinical signs of pneumonia were examined using thoracic ultrasonography, and foals with areas of pulmonary abscessation or consolidation were subjected to tracheobronchial aspiration for cytologic examination and microbiologic culture of aspirate fluid. A foal was considered to have clinical $R$. equi pneumonia when it had clinical signs of pneumonia, ultrasonographic evidence of pulmonary abscesses or consolidation, and a positive culture of $R$. equi from a tracheobronchial aspirate with cytological evidence of septic inflammation [15].

For the current study, 12 horses were randomly selected from 51 horses remaining at the 6666 Ranch that were part of the source population used in 2011. Using methods described previously, [15] TRPM2 genotypes were then determined for these 12 horses (AA, $n=3$; $\mathrm{AB}, n=4$; and $\mathrm{BB} n=5$ ). This sample size was determined by the funding available for this project and the cost for RNA-Seq.

\section{Sample collection and RNA-Seq}

A whole blood sample $(5 \mathrm{~mL})$ was collected by jugular venipuncture into 2 Paxgene RNA Vacutainer tubes (PreAnalytiX, Hombrechtikon, Switzerland) at the 6666 Ranch, Guthrie, TX, June 25, 2014, to permit the RNA to be stabilized for transport to Texas A\&M University. Total RNA was isolated using the MagMax Paxgene RNA purification kit (Life Technologies). RNA-Sequencing libraries were generated using the TrueSeq RNA preparation kit (Illumina) with a polyA selection step. The samples were pooled and sequenced (125-base-pair [bp], paired-end sequencing) on 2 lanes of a HiSeq 2500 (Illumina) to account for lane artifacts. The Texas A\&M AgriLife Genomics and Bioinformatics core generated the RNA-Seq libraries and performed the RNA-Seq reactions. The raw data of the RNA-Seq reactions was processed using bioinformatic tools provided by the Texas A\&M Institute for Genome Sciences and Society (TIGSS). FASTQ reads were de-multiplexed and assessed for quality using FastQC. Duplicate samples were merged and aligned to the equine reference genome assembly (EquCab2) using Tophat (version 2.0.14) [21].

\section{Gene expression analysis for genotypic and phenotypic comparisons}

The primary aim of the study was to identify differentially expressed genes, using either a targeted (TRPM2 gene region, including isoforms) or untargeted (transcriptome-wide) approach, between horses with the TRPM2 AA genotype $(n=3)$ and horses with either the TRPM2 $\mathrm{AB}$ or $\mathrm{BB}$ genotypes $(n=9)$. The $\mathrm{AB}$ and $\mathrm{BB}$ 
TRPM2 genotypes were combined on the basis of our previous results indicating that the odds of disease were similar in comparisons between horses with the AA TRPM2 genotype relative to the $\mathrm{AB}$ genotype $(\mathrm{OR}=4.3$; $P=0.0017)$, the $\mathrm{BB}$ genotype $(\mathrm{OR}=3.6 ; P=0.0574)$, or combined genotypes $(\mathrm{OR}=3.7 ; P=0.0006)$ [15]. A secondary aim was to compare differential gene expression between horses that had developed $R$. equi pneumonia as foals $(n=2)$ and horses that had not developed clinical signs of pneumonia as foals (i.e., subclinical pneumonia or no pneumonia; $n=10$ ), independent of TRPM2 genotype.

Sequencing reads were aligned to the equine genome using Tophat with the default parameters. EdgeR, which operates within the $\mathrm{R}$ statistical package (Version 3.0.1; $\mathrm{R}$ Statistical Project), and cuffdiff were used to identify differentially expressed genes using the Ensembl equine gene annotation [build 83] [21-26]. For the edgeR analysis, read counts of each gene were first determined using HTSeq [27] with the intersection nonempty parameter to account for ambiguous reads. The resulting count table was filtered to remove genes in which 0 reads mapped in all samples. The tabulated read counts were then normalized relative to library size and tag-wise and common dispersions estimated. Differentially expressed genes were defined as those genes having a false discovery rate $(\mathrm{FDR}) \leq 0.05$ that were identified with the exactTest function. For the cuffdiff analysis the default settings were used with the exception of the minimum isoform fraction setting $(F-0)$. Differentially expressed genes were defined as those having a q-value (FDR) $\leq 0.05$.

Biological pathway analysis was performed using the Ingenuity Pathway Analysis (IPA) (Qiagen, Venlo, Netherlands; www.ingenuity.com) tool-kit. Output files from both edgeR and cuffdiff were used in their respective pathway analyses. Input files for IPA used the following information from the edgeR or cuffdiff outputs: a column containing an Ensembl gene identifier, a corresponding gene name where applicable, the log fold-change of expression for each gene between the 2 groups tested, and a FDR for each gene tested.

Visualization of RNA-Seq read coverage across the region of interest was carried out using BEDTools and the University of California Santa Cruz (UCSC) online genome browser using aligned reads from the previously described Tophat mapping step [28, 29]. The cufflinks function of the Tophat tool suite was used to identify novel transcripts at the TRPM2 locus [21]. The Ensembl annotated TRPM2 gene model was then replaced with the TRPM2 assembled transcripts generated through Cufflinks. Analysis of the TRPM2 isoforms was performed using cuffdiff.

The assembled TRPM2 transcripts were verified by polymerase chain reaction (PCR). Briefly, equine RNA was isolated using the Ambion PureLink ${ }^{\mathrm{TM}}$ RNA isolation kit (Ambion, Waltham, MA) and cDNA synthesized using the iScript $^{\mathrm{TM}}$ cDNA synthesis kit (BIO-RAD, Hercules, CA). PCR probes were developed using the Primer3 software [30] for the $3^{\prime}$ end of predicted TRPM-1 with complementary reverse primers targeted at the $5^{\prime}$ and $3^{\prime}$ ends of TRPM2-2. Cycling conditions were as follows: $94{ }^{\circ} \mathrm{C}$ for $2 \mathrm{~min}, 94{ }^{\circ} \mathrm{C}$ for $30 \mathrm{~s}$ to $58{ }^{\circ} \mathrm{C}$ for $45 \mathrm{~s}$ to $72{ }^{\circ} \mathrm{C}$ for $1 \mathrm{~min}$ 30 times, followed by $72{ }^{\circ} \mathrm{C}$ for $3 \mathrm{~min}$. PCR products were resolved on a $1 \%$ agarose gel.

\section{Results}

RNA-seq of horse samples

RNA-seq generated an average of 33.7 million paired-end reads per sample of which $78.7 \%$ (viz., 26.5 million) uniquely aligned to the equine genome. With the exception of 1 sample ( $v i z$., foal 45), all samples had base quality scores $($ Phred) $>30$.

\section{Analysis of TRPM2 region gene expression and assembled transcripts (targeted approach)}

Neither the TRPM2 nor adjacent genes were differentially expressed between the AA and non-AA genotypes. Visualization of the aligned RNA-Seq reads relative to the TRPM2 gene annotation (Ensembl equine 83 and Non-horse RefSeq annotation) suggested that there were unnanotated exons and 3' untranslated regions (UTR) of the TRPM2 gene expressed in leukocytes (Fig. 1a). This observation promoted us to ask whether the TRPM2 isoforms were differentially expressed between horses with different TRPM2 genotypes as described above. To test this, we first merged the transcriptomes of each animal to increase the total depth of coverage of the transcriptomes and used Cufflinks to assemble transcripts of TRPM2. We then examined differential expression of the assembled TRPM2 transcripts as described above. The transcript assembly revealed 2 unspliced $5^{\prime}$ and $3^{\prime}$ transcripts of TRPM2 (hereafter referred to as TRPM2-1 and TRPM2-2) that included 17 novel isoforms (TRPM2-1 = 10, TRPM2$2=7$ ) consisting of unnanotated exons (e.g., $5^{\prime}$ terminal and cassette exons), retained introns, and 3' UTRs (Fig. 1b). Neither TRPM2-1 nor TRPM2-2 were differentially expressed between the 2 cohorts (edgeR and cuffdiff). Likewise, no differentially expressed isoforms were identified between the 2 groups. We then examined differential expression of the TRPM2 transcripts among the 3 TRPM2 genotypes (AA vs $\mathrm{AB}$; $\mathrm{AA}$ vs $\mathrm{BB} ; \mathrm{AB}$ vs $\mathrm{BB}$ ). The TRPM21 transcript was significantly higher in the $A B$ group in comparison to the AA group as well as higher than AA in all comparisons (Table 1).

To determine whether the 2 predicted genes at the annotated at TRPM2 locus are in fact 2 independent transcriptional units, we isolated RNA, synthesized cDNA, and probed with primers targeted for the $3^{\prime}$ end of TRPM2-1 and the $5^{\prime}$ end of TRPM-2. Gel electrophoresis 


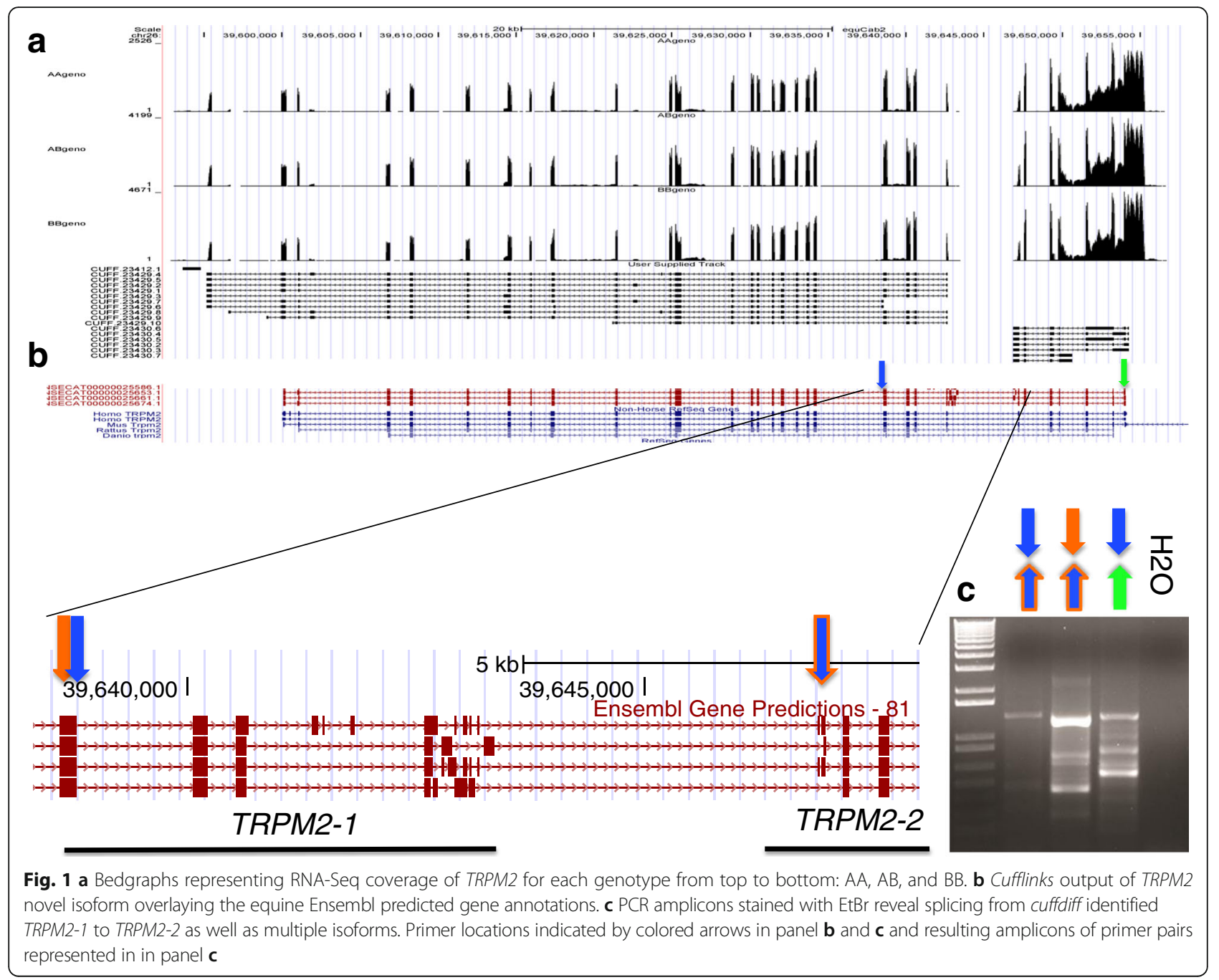

Table 1 Pairwise comparisons of potentially novel TRPM2 transcript expression values

\begin{tabular}{|c|c|c|c|c|c|c|}
\hline Cufflinks ID & Transcript ID & AA & $A B / B B$ & $\log _{2} F C$ & $P$ value & $P$ adjusted \\
\hline CUFF.23429 & TRPM2-1 & 90.99 & 118.90 & 0.39 & 0.0971 & 0.1942 \\
\hline \multirow[t]{2}{*}{ CUFF.23430 } & TRPM2-2 & 168.23 & 214.17 & 0.35 & 0.1630 & 0.3260 \\
\hline & & AA & $A B$ & & & \\
\hline CUFF.23429 & TRPM2-1 & 91.81 & 130.55 & 0.51 & 0.0224 & 0.0448 \\
\hline \multirow[t]{2}{*}{ CUFF.23430 } & TRPM2-2 & 169.73 & 225.94 & 0.41 & 0.0783 & 0.1566 \\
\hline & & $A A$ & BB & & & \\
\hline CUFF.23429 & TRPM2-1 & 90.63 & 110.04 & 0.28 & 0.2663 & 0.5326 \\
\hline \multirow[t]{2}{*}{ CUFF.23430 } & TRPM2-2 & 167.57 & 205.44 & 0.29 & 0.2766 & 0.5532 \\
\hline & & $A B$ & BB & & & \\
\hline CUFF.23429 & TRPM2-1 & 128.83 & 110.27 & -0.22 & 0.3456 & 0.6912 \\
\hline CUFF.23430 & TRPM2-2 & 223.02 & 205.87 & -0.12 & 0.6565 & 1.0000 \\
\hline
\end{tabular}


of the amplicons revealed that PCR successfully amplified transcripts spanning the region, indicating that TRPM2-1 and -2 are, at least in some instances, spliced together as a single transcription unit (Fig. 1c). Visual inspection of the PCR gel revealed several splice variants (amplicons) synthesized between the primer sets which corresponds with the several exons predicted by the Ensembl gene prediction track. This analysis does not rule out the possibility that independent transcriptional units might be synthesized from the TRPM2-1 and -2 locus.

\section{Differential gene expression analysis of transcriptome (untargeted approach)}

Examination of the gene count tables generated by HTSeq revealed 8212 genes with $<1$ read count/gene, leaving 18,779 genes for analysis in edgeR. Comparison of the TRPM2 genotypes (AA $[n=3]$ vs. $\mathrm{AB}[n=4]+\mathrm{BB}$ $[n=5])$ showed that expression of the ankyrin repeat domain 22 (ANKRD22), major histocompatibility complex, class II, DQ beta $(D Q B)$, and myeloperoxidase (MPO) genes were significantly higher $(\mathrm{FDR} \leq 0.05)$ in horses with the TRPM2 AA genotype compared to horses with the TRPRM2 AB and $\mathrm{BB}$ genotypes (Table 2). Cuffdiff analysis revealed 58 differentially expressed genes between the 2 groups (Table 3); however, none of the genes were concordant between the 2 analyses. Although not significantly different, expression of $D Q B$ was closest to being differentially expressed $(P=0.00075, \mathrm{FDR}=0.108)$ in the cuffdiff analysis, with the ANKRD22 and MPO having FDRs of 0.73 and 0.63 , respectively. Pathway analysis of the differentially expressed genes identified by cuffdiff revealed biological processes involving host antimicrobial and inflammatory response (17 genes), cell-tocell signaling, and cell interaction (Fig. 2). Notably, the CCAAT/enhancer binding protein epsilon $(C / E B P E)$, which was a central gene in the identified pathways, was expressed $\sim 1.3$-fold higher in the horses with TRPM2
AA genotypes relative to the other horses. No biological processes were identified using the differentially expressed genes identified by edgeR. Comparison of transcriptome by clinical status as foals (i.e., $R$. equi pneumonia $[n=2]$, unaffected $[n=10])$ using the analysis methods described above identified no differentially expressed genes (FDR $\leq 0.05)$.

\section{Discussion}

As previously reported, TRPM2 markers have been associated with clinical disease caused by $R$. equi [15]. The TRPM2 gene was biologically plausible as a candidate gene because TRPM2 has been shown to increase tissue damage at sites of inflammation in a mouse model of ulcerative colitis $[15,31]$. On the basis of these findings, we considered it important to understand the expression pattern across the region of interest identified by our previous GWAS (targeted approach). We found evidence indicating that alternative splicing occurs within the TRPM2 locus in horses resulting in multiple isoforms. Although we did not attempt to verify the functionality of these transcripts in horses, an alternative transcript has been functionally characterized in humanderived cell lines: a short isoform of TRPM2 inhibits calcium influx while increasing cell viability [32]. Given our demonstration of only a single TRPM2 gene in the equine genome, it is biologically plausible that the short isoforms of TRPM2 in the horse might have altered function that could confer some degree of protection against infection and subsequent tissue damage. Further experimentation is required to understand the function of these isoforms of equine TRPM2 and what role these isoforms might play in innate immune responses to $R$. equi infection.

The $C / E B P$ gene was identified as differentially expressed by cuffdiff (Table 3) and was indirectly implicated by IPA (Fig. 2). Like TRPM2, this gene is associated with neutrophil

Table 2 Transcriptome-wide differentially expressed genes identified by edge $R$ analysis

\begin{tabular}{|c|c|c|c|c|c|}
\hline Ensembl ID & Gene name & $\log F C^{a}$ & $\log _{C P M}{ }^{b}$ & $P$ value & FDR \\
\hline ENSECAG00000022239 & ANKRD22 & -5.82 & 0.23 & 0.0000 & 0.0000 \\
\hline ENSECAG00000006492 & $\mathrm{DQB}$ & -2.00 & 7.30 & 0.0000 & 0.0028 \\
\hline ENSECAG00000006662 & MPO & -3.22 & -0.44 & 0.0000 & 0.0483 \\
\hline ENSECAG00000017147 & C15orf52 & -3.08 & -1.66 & 0.0000 & 0.0968 \\
\hline ENSECAG00000002249 & PLEKHG4B & -4.64 & -1.57 & 0.0000 & 0.0983 \\
\hline ENSECAG00000008171 & N/A & -3.80 & -0.68 & 0.0000 & 0.1476 \\
\hline ENSECAG00000008238 & S100A5 & -3.41 & -1.98 & 0.0001 & 0.1476 \\
\hline ENSECAG00000016666 & OMG & 0.89 & 6.37 & 0.0001 & 0.2650 \\
\hline ENSECAG00000006656 & N/A & -3.08 & -2.89 & 0.0002 & 0.4258 \\
\hline ENSECAG00000024043 & CSTA & -2.09 & 2.50 & 0.0003 & 0.4904 \\
\hline
\end{tabular}


Table 3 Transcriptome-wide differentially expressed genes identified by cuffdiff analysis

\begin{tabular}{|c|c|c|c|c|c|}
\hline Ensembl ID & Gene name & $\log _{2} F C$ & $P$ value & Q value & Significant \\
\hline ENSECAG000000024043 & CSTA & -2.08 & 0.0001 & 0.0122 & yes \\
\hline ENSECAG00000024259 & DQA & -1.83 & 0.0001 & 0.0122 & yes \\
\hline ENSECAG00000019922 & ADAMDEC1 & -1.39 & 0.0001 & 0.0122 & yes \\
\hline ENSECAG00000009142 & DQA & -1.34 & 0.0001 & 0.0122 & yes \\
\hline ENSECAG00000020816 & PDLIM1 & -1.32 & 0.0001 & 0.0122 & yes \\
\hline ENSECAG00000015109 & $\mathrm{N} / \mathrm{A}$ & -1.28 & 0.0001 & 0.0122 & yes \\
\hline ENSECAG00000012883 & CEBPE & -1.27 & 0.0001 & 0.0122 & yes \\
\hline ENSECAG00000023062 & N/A & -1.26 & 0.0001 & 0.0122 & yes \\
\hline ENSECAG00000019130 & SIRPG & -1.22 & 0.0001 & 0.0122 & yes \\
\hline ENSECAG00000011315 & EMR3 & -1.17 & 0.0001 & 0.0122 & yes \\
\hline ENSECAG00000013660 & C1orf186 & -1.16 & 0.0002 & 0.0371 & yes \\
\hline ENSECAG00000025078 & SUSD2 & -1.05 & 0.0001 & 0.0122 & yes \\
\hline ENSECAG00000016730 & CSMD1 & -0.99 & 0.0001 & 0.0122 & yes \\
\hline ENSECAG00000001282 & CCR3 & -0.95 & 0.0001 & 0.0122 & yes \\
\hline ENSECAG00000008322 & GZMH & 0.79 & 0.0002 & 0.0371 & yes \\
\hline ENSECAG000000007621 & TRIP11 & 0.86 & 0.0003 & 0.0435 & yes \\
\hline ENSECAG00000018564 & SPATS2L & 0.87 & 0.0001 & 0.0221 & yes \\
\hline ENSECAG00000019111 & CD163 & 0.89 & 0.0002 & 0.0308 & yes \\
\hline ENSECAG000000020763 & MGST1 & 0.91 & 0.0001 & 0.0221 & yes \\
\hline ENSECAG00000014422 & OAS2 & 0.92 & 0.0001 & 0.0122 & yes \\
\hline ENSECAG00000011776 & MX1 & 0.93 & 0.0001 & 0.0221 & yes \\
\hline ENSECAG00000021989 & DDX58 & 0.94 & 0.0002 & 0.0308 & yes \\
\hline ENSECAG00000003474 & TTLL3 & 0.94 & 0.0001 & 0.0122 & yes \\
\hline ENSECAG00000001399 & SAMD9 & 1.03 & 0.0001 & 0.0221 & yes \\
\hline ENSECAG00000014218 & SIGLEC1 & 1.04 & 0.0003 & 0.0435 & yes \\
\hline ENSECAG00000008274 & CLEC4E & 1.05 & 0.0001 & 0.0122 & yes \\
\hline ENSECAG00000010117 & S100A9 & 1.05 & 0.0001 & 0.0122 & yes \\
\hline ENSECAG00000013762 & NCR1 & 1.07 & 0.0002 & 0.0308 & yes \\
\hline ENSECAG000000023733 & MMP-1 & 1.10 & 0.0001 & 0.0221 & yes \\
\hline ENSECAG00000015006 & FGFR1 & 1.10 & 0.0003 & 0.0435 & yes \\
\hline ENSECAG00000007133 & TPPP3 & 1.11 & 0.0001 & 0.0122 & yes \\
\hline ENSECAG00000022042 & PNP & 1.11 & 0.0001 & 0.0122 & yes \\
\hline ENSECAG00000008356 & ZNF577 & 1.15 & 0.0003 & 0.0435 & yes \\
\hline ENSECAG00000012235 & $\mathrm{BAZ2B}$ & 1.15 & 0.0001 & 0.0122 & yes \\
\hline ENSECAG00000009742 & $\mathrm{S} 100 \mathrm{~A} 12$ & 1.15 & 0.0001 & 0.0122 & yes \\
\hline ENSECAG00000021476 & MMP8 & 1.16 & 0.0001 & 0.0122 & yes \\
\hline ENSECAG00000009271 & S100A8 & 1.18 & 0.0001 & 0.0122 & yes \\
\hline ENSECAG00000019411 & HERC6 & 1.20 & 0.0001 & 0.0122 & yes \\
\hline ENSECAG00000026820 & SEPP1 & 1.22 & 0.0001 & 0.0122 & yes \\
\hline ENSECAG00000007881 & $\mathrm{IFIH1}$ & 1.24 & 0.0001 & 0.0122 & yes \\
\hline ENSECAG00000014645 & OASL & 1.29 & 0.0001 & 0.0122 & yes \\
\hline ENSECAG00000010153 & IFIT4 & 1.33 & 0.0001 & 0.0122 & yes \\
\hline ENSECAG00000013874 & SPARC & 1.44 & 0.0002 & 0.0308 & yes \\
\hline ENSECAG000000001481 & SAMD9L & 1.47 & 0.0001 & 0.0122 & yes \\
\hline
\end{tabular}


Table 3 Transcriptome-wide differentially expressed genes identified by cuffdiff analysis (Continued)

\begin{tabular}{|c|c|c|c|c|c|}
\hline ENSECAG00000008809 & OAS3 & 1.51 & 0.0001 & 0.0122 & yes \\
\hline ENSECAG00000000500 & IF16 & 1.53 & 0.0001 & 0.0122 & yes \\
\hline ENSECAG00000015395 & HERC5 & 1.54 & 0.0001 & 0.0122 & yes \\
\hline ENSECAG00000001324 & ISG15 & 1.55 & 0.0001 & 0.0122 & yes \\
\hline ENSECAG00000008594 & BTN3A1 & 1.59 & 0.0001 & 0.0122 & yes \\
\hline ENSECAG00000006913 & N/A & 1.90 & 0.0001 & 0.0122 & yes \\
\hline ENSECAG00000019949 & CYP4F & 1.92 & 0.0001 & 0.0122 & yes \\
\hline ENSECAG00000017437 & MYLPF & 2.38 & 0.0002 & 0.0371 & yes \\
\hline ENSECAG00000004349 & IFIT5 & 2.54 & 0.0001 & 0.0122 & yes \\
\hline ENSECAG00000020407 & MYBPC2 & 2.64 & 0.0002 & 0.0371 & yes \\
\hline ENSECAG00000010020 & $\mathrm{HBB}$ & 2.73 & 0.0001 & 0.0122 & yes \\
\hline ENSECAG00000023971 & TNNT3 & 4.46 & 0.0002 & 0.0371 & yes \\
\hline ENSECAG00000019728 & TNNC2 & 5.11 & 0.0001 & 0.0122 & yes \\
\hline ENSECAG00000005487 & N/A & 8.08 & 0.0001 & 0.0122 & yes \\
\hline
\end{tabular}

function. Isoforms of the $C / E B P$ gene are expressed by human neutrophils and play an integral part in inducing several inflammatory cytokines [33]. C/EBP was shown to bind the promoter of interleukin 8 (IL-8, CXCL8) when stimulated by lipopolysaccharides (LPS), [33] and upregulation of CXCL8 production by TRPM2 has been linked to increased tissue damage at sites of inflammation in mice [31, 34].

The transcriptome-wide analysis for association of gene expression with TRPM2 SNP genotype using both edgeR and cuffdiff did not reveal any concordant results. This might be attributable to several factors. Each program uses a different method to predict differentially expressed genes and each is tailored to conduct different types of analysis. Cuffdiff uses a mixture of distributions to account for the uncertainty in mapping a read and the variability in read count while edgeR primarily focuses on the variability in read count across replicates $[21,22]$. The results from edgeR appear to be more conservative in our experiment because only 3 genes were identified as differentially expressed whereas cuffdiff identified 58 genes. There is also evidence to suggest that edgeR is not always a more conservative approach;

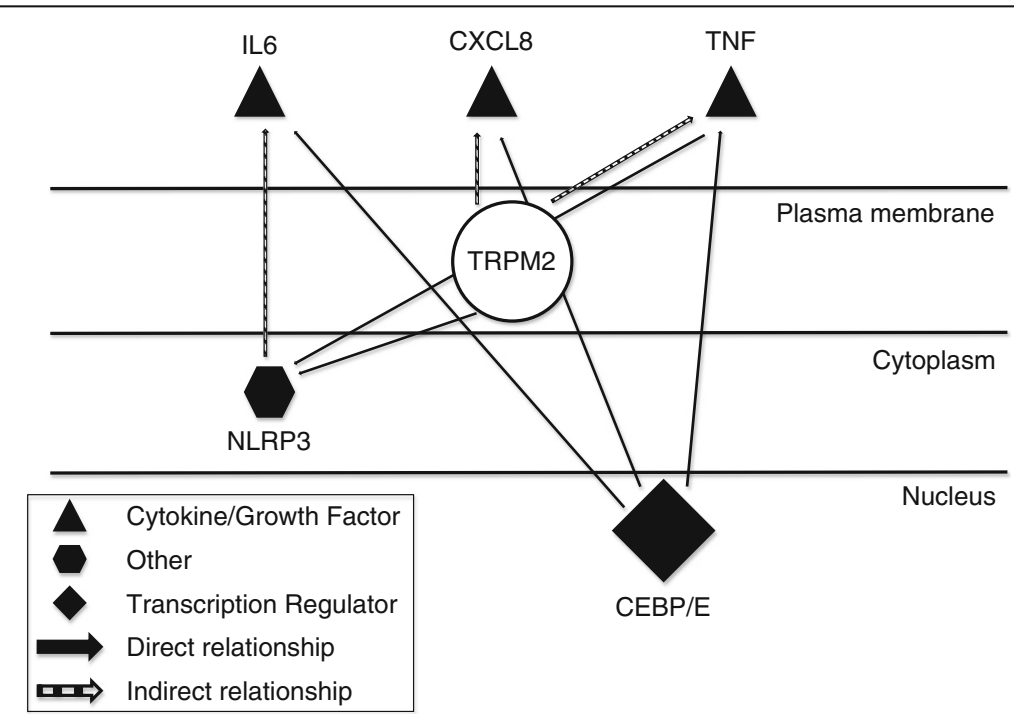

Fig. 2 EdgeR pathway analysis results. TRPM2 was not identified as differentially expressed in the analysis but was added for the purpose of identifying TRPM2's link with the differentially expressed genes. Solid arrows indicate a documented direct interaction, while dashed arrows represent interactions linked through an intermediary. The transcription regulator CEBP/E directly interacts with IL6, CXCL8, and TNF establishing a link between the homozygous AA TRPM2 horses and expression of immune related genes. The indirect interactions show the relationship of TRPM2 to the differentially expressed genes and thus suggesting a role for TRPM2 in innate immunity. Figure adapted from IPA generated output for resolution purposes 
thus, the observed discrepancy might reflect some other biological or technical property present in the data but no other metrics suggest this was the case [35]. Also, cuffdiff uses the alignments from TopHat and a gene annotation file to calculate differential expression. This removes the HTSeq count step and might have contributed to the differing results. In an effort to maximize the inferences drawn from our data, we elected to use the genes identified by cuffdiff to better leverage our data in elucidating biological pathways and processes playing a role in susceptibility to $R$. equi pneumonia.

There are several other factors to consider regarding RNA-Seq and the outcome of its analysis. RNA-Seq reflects steady-state levels of RNA, which encompasses the rate of transcription, rate of degradation, post-transcriptional phases, and post-translational modifications that may alter protein function [36]. For example, a 1.3-fold increase in expression of $C / E B P$ within the AA genotypes group over the non-AA genotypes is a complex finding, as it is in any case investigating differentially expressed genes. A first step in following up on the findings and towards a better understanding this gene and pathway as it relates to $R$. equi pneumonia would be to confirm the RNA expressions levels by another method. Also, we do not know whether a 1.3-fold increase in RNA represents a 1.3-fold increase in the protein level of C/EBP in these horses. A recent study found that on average only $40 \%$ of the variability in protein levels of the cell could be explained by mRNA levels [37]. This finding demonstrates that the dynamic processes of transcription and translation cannot be easily generalized to their functional implications.

Our transcriptome-wide analysis of gene expression with disease status compared the samples based on a horse's phenotypic classification when it was a foal. No evidence that disease status as a foal was associated with gene expression as an adult; however, our study was clearly lacking power and results of absence of evidence of an association should not be confused with evidence of absence of an association.

Our study had a number of limitations. This project was conducted using mature horses and not foals: gene expression as adults might not reflect the gene expression of these horses when they were foals and susceptible to $R$. equi. The marker genotypes (i.e., $\mathrm{AA}, \mathrm{AB}$, and $\mathrm{BB}$ ), however, would not have changed and therefore their impact on functional transcription should remain the same. A second limitation is that the region of interest's involvement with and relationship to $R$. equi infection might be tissue-specific (e.g., in the lung or in alveolar macrophages) and thus not captured or accurately reflected by RNA-Seq data from whole blood. A third limitation is the small sample size of the study which was dictated by available funding. This was particularly true for our secondary aim comparing gene expression by pneumonia status as a foal. Nevertheless, previous studies have yielded important results using fewer horses than this and our results provide novel, significant findings relevant to understanding the pathogenesis and susceptibility of foals to $R$. equi infection [18-20].

To the authors' knowledge, these data represent the first whole transcriptome assembly of the Quarter Horse genome. With an average of 33.7 million reads per sample, we have generated an average of $53.83 \mathrm{X}$ coverage of the Quarter Horse transcriptome. The cufflinks program estimated over 34,000 genes to be present in the Quarter Horse genome. Furthermore, cufflinks estimated over 74,500 isoforms of these 34,000 genes to be transcribed. The RNA-Seq data presented in this report should be valuable to those interested in the equine transcriptome because researchers will be able to align their transcriptomic/genomic data with the Quarter Horse transcriptome to further investigate functional implications of comparative genomics.

\section{Conclusions}

Rhodococcus equi is a pathogen that predominantly affects young foals with often severe and potentially fatal outcomes. Here we described several differentially expressed genes, identified using RNA-Seq, associated with this genotype that are important to innate immune responses. Specifically, $C / E B P$ was found to be upregulated in horses with the susceptible genotype. This is an important finding due to the role $C / E B P$ plays in enhancing $I L-8$ expression as increased concentration of $I L-8$ at sites of inflammation has been shown to increase tissue damage. These findings suggest that modulation of expression of TRPM2 contributes to susceptibility to $R$. equi pneumonia and has shed further light on our understanding of the susceptibility genotype and its relationship to gene expression.

\section{Abbreviations \\ ANKRD22: Ankyrin repeat domain 22; Bp: Base-pair; C/EBPE: CCAAT/enhancer binding protein epsilon; DQB: Major histocompatibility complex, class II, DQ beta; FDR: False discovery rate; $\mathrm{Fe}^{2+}$ : Iron; GWAS: Genome-wide association study; IL-8, CXCL8: Interleukin 8; IPA: Ingenuity Pathway Analysis; LPS: Lipopolysaccharide; MPO: Myeloperoxidase; PCR: Polymerase chain reaction; PE: Paired-end; $R$. equi: Rhodococcus equi; RNA-Seq: RNA-Sequencing; SNP: Single nucleotide polymorphism; TRPM2: Transient receptor potential cation channel, subfamily M, member 2; UCSC: University of California, Santa Cruz; UTR: Untranslated region; VapA: Virulence associated protein}

\section{Acknowledgements}

We would like to thank the Texas A\&M Institute for Genome Sciences and Society for providing computing resources. Additionally, we would like to thank the 6666 Ranch for their support and commitment to providing horses for this study.

\section{Funding}

This work was funded through a grant from the Department of Large Animal Clinical Sciences at Texas A\&M University, with additional support provided by the Link Equine Research Endowment. Also, Cole McQueen (CMM) was supported by a grant from the USDA-NIFA \#2011-68004-30367. 


\section{Availability of data and materials}

All RNA-Seq data have been deposited in NCBI's Gene Expression Omnibus (GEO) [38] and are accessible through GEO Series accession number GSE81858 (http://www.ncbi.nlm.nih.gov/geo/query/acc.cgi?acc=GSE81858).

\section{Authors' contributions}

NDC, SVD, and CMM designed the study, performed sample collection, and drafted the manuscript. GPB provided permission for and access to samples. CMM processed samples. NDC, SVD, CMM, CWC, and KK analyzed and interpreted the data. All authors read and approved the final manuscript.

\section{Competing interests}

The authors declare that they have no competing interests.

\section{Consent for publication}

Not applicable.

\section{Ethics approval}

All protocols for this study were reviewed and approved by the Institutional Animal Care and Use Committee (AUP: IACUC 2013-0259 CA, Approval Date: 1/29/2014). Written informed consent for participation was obtained for all horses included in the study, and the 6666 Ranch provided access to the horses for use in this project.

\section{Author details}

'Department of Large Animal Clinical Sciences, Texas A\&M University College of Veterinary Medicine \& Biomedical Sciences, College Station, TX, USA. ${ }^{2}$ Texas A\&M Institute for Genome Sciences and Society, Texas A\&M University, College Station, TX, USA. ${ }^{36666}$ Ranch, 1102 Dash For Cash Road, Guthrie, TX, USA. ${ }^{4}$ Department of Veterinary Pathobiology, Texas A\&M University College of Veterinary Medicine \& Biomedical Sciences, College Station, TX, USA.

Received: 1 June 2016 Accepted: 25 November 2016 Published online: 05 December 2016

\section{References}

1. Prescott JF. Rhodococcus equil: an animal and human pathogen. Clin Microbiol Rev. 1991;4(1):20-34.

2. Chaffin MK, Cohen ND, Martens RJ. Evaluation of equine breeding farm characteristics as risk factors for development of Rhodococcus equi pneumonia in foals. J Am Vet Med Assoc. 2003;222(4):467-75.

3. Giguère $S$, Cohen ND, Keith Chaffin M, Slovis NM, Hondalus MK, Hines SA, Prescott JF. Diagnosis, treatment, control, and prevention of infections caused by Rhodococcus equi in foals. J Vet Intern Med. 2011;25(6):1209-20.

4. Giguere S, Cohen ND, Chaffin MK, Hines SA, Hondalus MK, Prescott JF, Slovis NM. Rhodococcus equi: clinical manifestations, virulence, and immunity. J Vet Intern Med. 2011;25(6):1221-30.

5. Chaffin MK, Cohen ND, Blodgett GP, et al. Evaluation of hematologic screening methods for predicting subsequent onset of clinically apparent Rhodococcus equi pneumonia in foals. Lexington: American Association of Equine Practitioners; 2013. p. 267.

6. Venner M, Reinhold B, Beyerbach M, Feige K. Efficacy of azithromycin in preventing pulmonary abscesses in foals. Vet J (London, England: 1997). 2009;179(2):301-3.

7. Giguere S, Hondalus MK, Yager JA, Darrah P, Mosser DM, Prescott JF. Role of the 85-kilobase plasmid and plasmid-encoded virulence-associated protein A in intracellular survival and virulence of Rhodococcus equi. Infect Immun. 1999;67(7):3548-57.

8. Jain S, Bloom BR, Hondalus MK. Deletion of vapA encoding virulence associated protein $\mathrm{A}$ attenuates the intracellular actinomycete Rhodococcus equi. Mol Microbiol. 2003;50(1):115-28.

9. Halbert ND, Cohen ND, Slovis NM, Faircloth J, Martens RJ. Variations in equid SLC11A1 (NRAMP1) genes and associations with Rhodococcus equi pneumonia in horses. J Vet Intern Med. 2006;20(4):974-9.

10. Horin P, Sabakova K, Futas J, Vychodilova L, Necesankova M. Immunity-related gene single nucleotide polymorphisms associated with Rhodococcus equi infection in foals. Int J Immunogenet. 2010;37(2):67-71.

11. Mousel MR, Harrison L, Donahue JM, Bailey E. Rhodococcus equi and genetic susceptibility: assessing transferrin genotypes from paraffin-embedded tissues. J Vet Diagn Investig. 2003;15(5):470-2.
12. Horin P, Smola J, Matiasovic J, Vyskocil M, Lukeszova L, Tomanova K, Kralik P, Glasnak V, Schroffelova D, Knoll A, et al. Polymorphisms in equine immune response genes and their associations with infections. Mamm Genome. 2004:15(10):843-50.

13. McQueen CM, Dindot SV, Foster MJ, Cohen ND. Genetic susceptibility to Rhodococcus equi. J Vet Intern Med. 2015;29:1648-1659.

14. Tabor HK, Risch NJ, Myers RM. Candidate-gene approaches for studying complex genetic traits: practical considerations. Nat Rev Genet. 2002;3(5):391-7.

15. McQueen CM, Doan R, Dindot SV, Bourquin JR, Zlatev ZZ, Chaffin MK, Blodgett GP, Ivanov I, Cohen ND. Identification of genomic loci associated with Rhodococcus equi susceptibility in foals. PLoS One. 2014;9(6):e98710.

16. McCue ME, Bannasch DL, Petersen JL, Gurr J, Bailey E, Binns MM, Distl O, Guérin G, Hasegawa T, Hill EW, et al. A high density SNP array for the domestic horse and extant Perissodactyla: utility for association mapping, genetic diversity, and phylogeny studies. PLoS Genet. 2012;8(1):e1002451.

17. Wang Z, Gerstein M, Snyder M. RNA-Seq: a revolutionary tool for transcriptomics. Nat Rev Genet. 2009;10(1):57-63.

18. Park KD, Park J, Ko J, Kim BC, Kim HS, Ahn K, Do KT, Choi H, Kim HM, Song $\mathrm{S}$, et al. Whole transcriptome analyses of six thoroughbred horses before and after exercise using RNA-Seq. BMC Genomics. 2012;13:473.

19. Coleman SJ, Zeng Z, Wang K, Luo S, Khrebtukova I, Mienaltowski MJ, Schroth GP, Liu J, MacLeod JN. Structural annotation of equine protein-coding genes determined by mRNA sequencing. Anim Genet. 2010;41 Suppl 2:121-30.

20. Capomaccio S, Vitulo N, Verini-Supplizi A, Barcaccia G, Albiero A, D'Angelo M, Campagna D, Valle G, Felicetti M, Silvestrelli M, et al. RNA sequencing of the exercise transcriptome in equine athletes. PLoS One. 2013;8(12):e83504.

21. Trapnell C, Roberts A, Goff L, Pertea G, Kim D, Kelley DR, Pimentel H, Salzberg $S L$, Rinn JL, Pachter L. Differential gene and transcript expression analysis of RNA-seq experiments with TopHat and Cufflinks. Nat Protoc. 2012;7(3):562-78.

22. Robinson MD, McCarthy DJ, Smyth GK. edgeR: a Bioconductor package for differential expression analysis of digital gene expression data. Bioinformatics (Oxford, England). 2010;26(1):139-40.

23. McCarthy DJ, Chen Y, Smyth GK. Differential expression analysis of multifactor RNA-Seq experiments with respect to biological variation. Nucleic Acids Res. 2012;40(10):4288-97.

24. Robinson MD, Smyth GK. Moderated statistical tests for assessing differences in tag abundance. Bioinformatics (Oxford, England). 2007;23(21):2881-7.

25. Robinson MD, Smyth GK. Small-sample estimation of negative binomial dispersion, with applications to SAGE data. Biostatistics. 2008;9(2):321-32.

26. Zhou X, Lindsay H, Robinson MD. Robustly detecting differential expression in RNA sequencing data using observation weights. Nucleic Acids Res. 2014; 42(11):e91.

27. Anders S, Pyl PT, Huber W. HTSeq-a Python framework to work with highthroughput sequencing data. Bioinformatics (Oxford, England). 2015;31(2):166-9.

28. Quinlan AR, Hall IM. BEDTools: a flexible suite of utilities for comparing genomic features. Bioinformatics (Oxford, England). 2010;26(6):841-2.

29. Kent WJ, Sugnet CW, Furey TS, Roskin KM, Pringle TH, Zahler AM, Haussler D. The human genome browser at UCSC. Genome Res. 2002;12(6):996-1006.

30. Untergasser A, Cutcutache I, Koressaar T, Ye J, Faircloth BC, Remm M, Rozen SG. Primer3-new capabilities and interfaces. Nucleic Acids Res. 2012;40(15):e115.

31. Yamamoto S, Shimizu S, Kiyonaka S, Takahashi N, Wajima T, Hara Y, Negoro T, Hiroi T, Kiuchi Y, Okada T, et al. TRPM2-mediated Ca2 + influx induces chemokine production in monocytes that aggravates inflammatory neutrophil infiltration. Nat Med. 2008;14(7):738-47.

32. Zhang W, Chu X, Tong Q, Cheung JY, Conrad K, Masker K, Miller BA. A novel TRPM2 isoform inhibits calcium influx and susceptibility to cell death. J Biol Chem. 2003;278(18):16222-9.

33. Cloutier A, Guindi C, Larivee P, Dubois CM, Amrani A, McDonald PP. Inflammatory cytokine production by human neutrophils involves C/EBP transcription factors. J Immunol (Baltimore, Md: 1950). 2009;182(1):563-71.

34. Shimizu S, Yonezawa R, Negoro T, Yamamoto S, Numata T, Ishii M, Mori Y, Toda T. Sensitization of HO-induced TRPM2 activation and subsequent interleukin-8 (CXCL8) production by intracellular Fe in human monocytic U937 cells. Int J Biochem Cell Biol. 2015;68:119-127.

35. Nookaew I, Papini M, Pornputtapong N, Scalcinati G, Fagerberg L, Uhlen M, Nielsen J. A comprehensive comparison of RNA-Seq-based transcriptome analysis from reads to differential gene expression and cross-comparison with microarrays: a case study in Saccharomyces cerevisiae. Nucleic Acids Res. 2012:40(20):10084-97.

36. Neymotin B, Athanasiadou R, Gresham D. Determination of in vivo RNA kinetics using RATE-seq. RNA (New York, NY). 2014;20(10):1645-52. 
37. Schwanhausser B, Busse D, Li N, Dittmar G, Schuchhardt J, Wolf J, Chen W, Selbach M. Global quantification of mammalian gene expression control. Nature. 2011;473(7347):337-42.

38. Edgar R, Domrachev M, Lash AE. Gene Expression Omnibus: NCBI gene expression and hybridization array data repository. Nucleic Acids Res. 2002:30(1):207-10

Submit your next manuscript to BioMed Central and we will help you at every step:

- We accept pre-submission inquiries

- Our selector tool helps you to find the most relevant journal

- We provide round the clock customer support

- Convenient online submission

- Thorough peer review

- Inclusion in PubMed and all major indexing services

- Maximum visibility for your research

Submit your manuscript at www.biomedcentral.com/submit 